\title{
Hemifacial Transplantation Model in Rats
}

Jong Woo Lim,

\section{Seok Chan Eun}

Department of Plastic and Reconstructive Surgery, Seoul National University Bundang Hospital, Seoul National University College of Medicine, Korea

No potential conflict of interest relevant to this article was reported.
Background: To refine facial transplantation techniques and achieve sound results, it is essential to develop a suitable animal model. Rat is a small animal and has many advantages over other animals that have been used as transplantation models. The purpose of this study was to describe a rat hemifacial transplantation model and to verify its convenience and reproducibility.

Methods: Animals used in this study were Lewis rats (recipients) and Lewis-Brown Norway rats (donors). Nine transplantations were performed, requiring 18 animals. The hemifacial flap that included the ipsilateral ear was harvested based on the unilateral common carotid artery and external jugular vein and was transferred as a single unit. Cyclosporine A therapy was initiated 24 hours after transplantation and lasted for 2 weeks. Signs of rejection responses were evaluated daily.

Results: The mean transplantation time was 1 hour 20 minutes. The anatomy of common carotid artery and external jugular vein was consistent, and the vessel size was appropriate for anastomosis. Six of nine allografts remained good viable without vascular problems at the conclusion of study (postoperative 2 weeks).

Conclusion: The rat hemifacial transplantation model is suitable as a standard transplantation training model.

Keywords: Rat / Facial transplantation / Vascularized composite allotransplantation

\section{INTRODUCTION}

Composite tissue allotransplantation (CTA) has a significant potential for application in the field of reconstructive surgery, with successful transplantation examples including the hand, abdominal wall, knee, flexor tendon apparatus, nerve, larynx, skeletal muscle, tongue, trachea, scalp, penis, and partial face transplants [1]. In the craniofacial reconstruction field, CTA could become an alternative to free autologous tissue transfer in patients severely disfigured as a result of trauma, such as burns, traffic accidents, animal bites, and gunshot wounds, as well as massive tumor re-

\section{Correspondence: Seok Chan Eun}

Department of Plastic and Reconstructive Surgery, Seoul National University Bundang Hospital, Seoul National University College of Medicine, 82 Gumi-ro, 173 Beon-gil, Bundang-gu, Sungnam 463-707, Korea

E-mail: sceun@snubh.org

*This research was supported by Basic Science Research Program through the National Research Foundation of Korea (NRF) funded by the Ministry of Education, Science and Technology (NRF-2012R1A1A2021731).

Received June 30, 2014 / Revised July 23, 2014 / Accepted August 1, 2014 sections. In practice, however, such transplantation faces various medical, administrative, social, ethical, and regulatory challenges [2]. Although many problems remain to be resolved, reconstructive surgeons should be prepared for a future in which CTA is the final option in the reconstruction ladder.

Availability of suitable animal models is essential for developing and improving surgical techniques and achieving successful transplantation in humans. Several animal facial transplantation models have been reported in the literature, including the rat, rabbit, dog, and primates [3-6]. The ideal facial transplantation experimental model should meet some important criteria, such as adequate animal size, anatomic variance, vessel pedicle consisten$c y$, reasonable cost and operation time, and ease of animal care $[3,4]$. The most critical two factors for success of CTA are revascularization of the donor flap by microanastomosis and prevention of rejection response with immunosuppressive therapy [2]. In this regard, a rat model is advantageous at initial stages of training be- 
cause the anatomy of common carotid artery and external jugular vein is consistent and the immune tolerance is well established [7]. The purpose of this study was to describe an experimental rat hemifacial transplantation model and to verify its convenience and reproducibility.

\section{METHODS}

\section{Experimental animals}

Animals used in this study were 6- to 10-week-old male Lewis and Lewis-Brown Norway rats weighing 180-220 g. Eighteen animals required for 9 transplantations were purchased from our local Laboratory Animal Breeding and Research Center. Lewis and Lewis-Brown Norway rats were used recipients and donors, respectively. All animals used in this study received humane care. They were kept warm with a light source and a heating pad and were maintained under specific pathogen-free conditions. All surgical procedures were performed under sterile conditions. Anesthesia was induced with sodium pentobarbital $(50 \mathrm{mg} / \mathrm{kg}$, administered intraperitoneally). Follow-up doses of $10 \mathrm{mg} / \mathrm{kg}$ were administered to maintain anesthesia after 1 hour. Head and neck were shaved, and the skin was thoroughly cleansed with $10 \%$ povidone-iodine solution. A prophylactic antibiotic (intramuscular potassium penicillin, 100,000 international units $/ \mathrm{kg}$ ) was administered before each operation. Lactated Ringer's solution was administered as a fluid supplement during the operation and on postoperative days 0 and 1 . After operation, all the recipients were caged individually under standard environmental conditions with a 12 hour light-dark cycle. Water and standard laboratory food were provided ad libitum. Postoperative analgesia was provided with buprenorphine $(0.5 \mathrm{mg} / \mathrm{kg}$, administered subcutaneously every 12 hours) for the first 2 days.

\section{Operative technique}

The donor hemifacial flap is designed on the left side (Fig. 1). Elliptical incisions were made around the eyes, leaving $1 \mathrm{~mm}$ of intact skin around the upper and lower eyelids. An additional incision

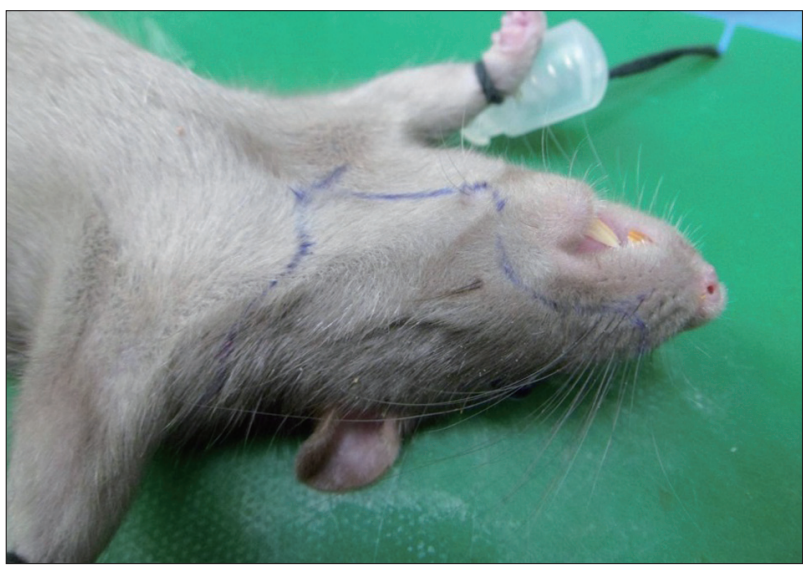

Fig. 1. Preoperative design of the donor hemifacial flap. The hemifacial flap was harvested from the left side of the Lewis-Brown Norway rats. The design landmarks are the mentum, suprasternal notch, oral commissure, nasal tip, and vertex.

was made $5 \mathrm{~mm}$ lateral to the nasal tip and extended periorally 3 $\mathrm{mm}$ apart from the oral commissure and the lower lip until the mentum. Next, a midline vertical incision from the mentum to the suprasternal notch was performed, extending deep to the platysmal layer. From the lower end of vertical incision, horizontal incisions were extended posteriorly until reaching the neck midline at the scalp hairline. Finally, a vertical incision from the vertex to the neck midline was made to complete the skin incision. In the neck, dissection was performed superiorly above the sternocleidomastoid muscle toward the angle of mandible, preserving the external jugular vein. Dissection was continued above the masseter muscle to the ear. Along the back of neck, the flap was elevated above the trapezius to the cartilaginous part of the external ear canal. The external ear was included in the flap after separation of the external ear canal. The sternocleidomastoid muscle was detached from its insertion and excised to expose the common carotid artery. Finally, the common carotid artery and external jugular vein were divided proximally to obtain adequately-long vascular pedicle to the flap (Fig. 2). The harvested hemifacial flap was perfused with heparinized lactated Ringer's solution until the venous outflow was clear.

The recipient site and vessels were prepared using a similar surgical method. The skin on the left side was removed with the exception of periorbital and perioral regions, which were left intact to preserve vital functions of the recipient after transplantation. 
The facial nerve and muscles were kept intact to avoid functional deformities that could interfere with feeding and breathing. Periauricular incisions were performed as described for the donor. The external jugular vein was isolated and prepared for anastomosis. The sternocleidomastoid muscle was retracted to expose the common carotid artery. The inferior half of the sternocleidomastoid muscle was excised for arterial end-to-side anastomosis (Fig. 3).

The harvested facial-scalp flap was transferred from the donor to recipient. The flap was sutured to the zygomaticus and masseter muscle fascia with absorbable sutures, which are 2 firmest regions of the facial flap that provides adequate tissue fixation to withstand postoperative gravitational and shearing forces. The vascular pedicles including common carotid arteries and external jugu- lar veins were approximated to their respective recipient vessels. Arterial end-to-end anastomosis was performed in the deeper tissue plane, and vein end-to-side anastomosis was performed in the superficial plane under an operative microscope (Zeiss OP-MI 6 SD, Carl Zeiss, Goettingen, Germany) using 10-0 nylon sutures. After skin closure, recipients received subcutaneous lactated Ringer's solution to compensate for perioperative fluid loss (Fig. 4). To facilitate recovery, animals were closely monitored during the postoperative period, with special attention paid to oral intake and adequate fluid supplementation. To prevent acute and chronic allograft rejection, cyclosporine A (Cipol, Chongkundang, Seoul, Korea) therapy was initiated 24 hours after transplantation and lasted for 2 weeks ( $16 \mathrm{mg} / \mathrm{kg}$ per day). Transplanted facial flaps were evaluated for signs of rejection on a daily basis.
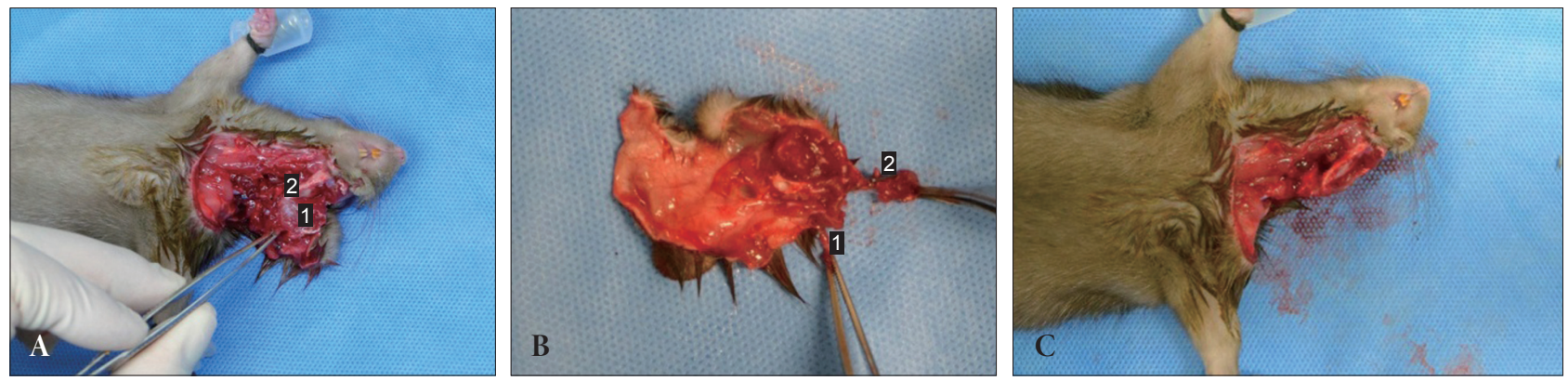

Fig. 2. An intraoperative photograph during harvesting of the donor composite tissue allograft. (A) Forceps indicate the left common carotid artery. 1, the masseter muscle; 2, the mandible angle. (B) The hemifacial composite tissue flap consists of skin, subcutaneous tissue, and ear with cartilage. 1, the common carotid artery; 2, the external jugular vein. (C) The donor after completion of the flap harvesting.

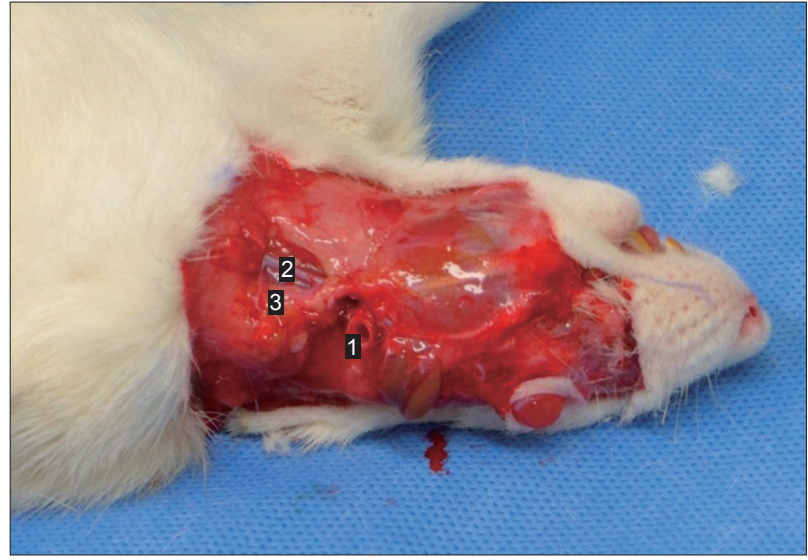

Fig. 3. A recipient after preparation for the flap transfer. Note that the periorbital and perioral skin of the Lewis rat is preserved. 1, the common carotid artery; 2, the external jugular vein; 3 , the sternocleidomastoid muscle.

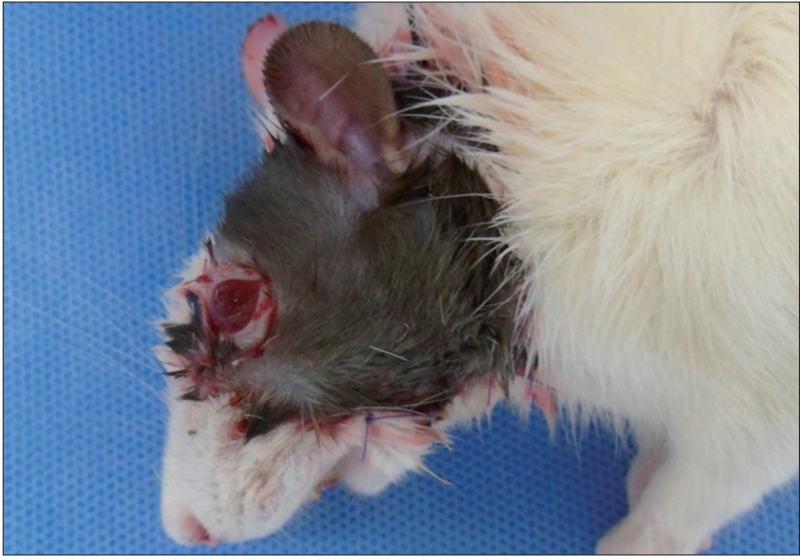

Fig. 4. An immediate postoperative photograph. 


\section{RESULTS}

The mean time required to accomplish the hemifacial transplantation procedure was 1 hour 20 minutes, and the mean warm ischemia time was 15 minutes. A successful outcome was achieved by using the distal part of the common carotid artery as recipient artery and the external jugular vein as recipient vein. The mean arterial diameter was $1.0 \mathrm{~mm}$ (range, $0.8-1.2 \mathrm{~mm}$ ), and the mean vein diameter was $2.1 \mathrm{~mm}$ (range, $1.8-2.4 \mathrm{~mm}$ ).

On post-transplantation day 1 , the animals returned to a normal routine of eating, drinking, and playing. Mild soft tissue edema and hematomas under the flaps were observed, but no cases required drainage. One allograft recipient died on day 2 under a poor general condition. Two recipients showed acute rejection signs on day 5 and were sacrificed by intravenous administration of $100 \mathrm{mg} / \mathrm{kg}$ sodium pentobarbital on day 7. The 6 remaining recipients showed no rejection response until the end of study period at 2 weeks after transplantation (Fig. 5).

\section{DISCUSSION}

Although composite face allotransplantation animal models have been studied by many plastic surgeons, there is a lack of agreement as to what species are the most suitable. The rat hemifacial transplantation model has many advantages. First, it is convenient in terms of performing the operation. Because the rat is a small

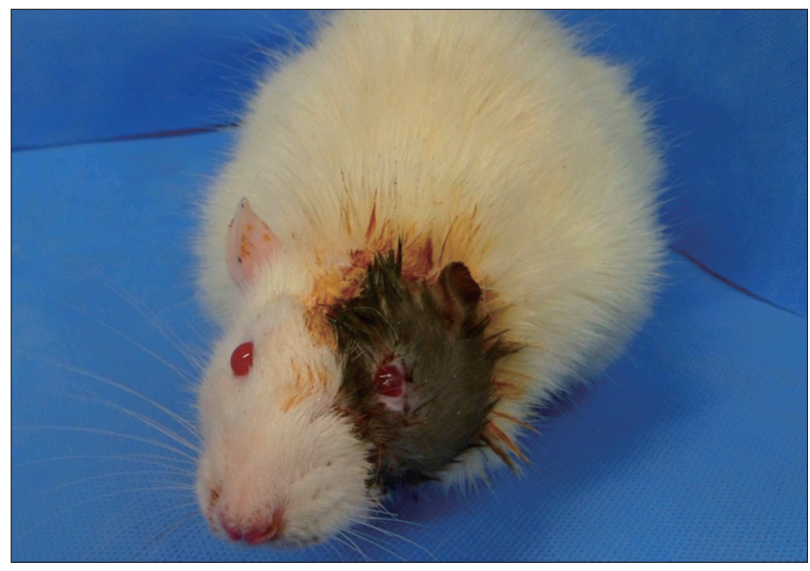

Fig. 5. A photograph on postoperative day 7. There were no acute rejection signs on clinical evaluation. animal, harvesting and insetting of the flap do not take much time, while the vessel size is still suitable for microanastomosis. In our study, the mean time of transplantation was only 1 hour 20 minutes, far shorter than the usually required in transplantation models that use larger animals such as rabbit, dog, and primates [4-6]. Second, the anatomy of composite tissue and blood vessels are consistent and comparable to those of the human. Third, preoperative preparation and anesthesia are simple. Fourth, the experiments are cost-effective because of the low cost of rats, food, and maintenance, when compared with transplantations performed in larger animals. Finally, the operation does not require advanced skills in microsurgery. As a result, this model can be implemented in many laboratories which previously had not been performing CTA.

Arterial and venous anastomosis are the most critical steps for flap survival and overall success of transplantation. In the present study, the common carotid arteries and external jugular veins of the donors and recipients were anastomosed in end-to-side and end-to-end manners, respectively. Initially introduced by Siemionow et al. [8], the full-face transplantation model in the rat involved the main vascular structures on both sides of the head and neck, and was associated with a longer operation time and higher perioperative mortality rates from brain ischemia and vascular complications [3,7]. In contrast, we have harvested flaps based on a unilateral common carotid artery and an external jugular vein, which had minimized complications related to brain ischemia. In fact, no such vascular complications were observed in our series of transplantation.

The order of flap inset and microanastomosis is debatable [9]. Although the significance of early revascularization of the harvested flap cannot be underestimated, it is technically difficult to fix the flap safely after performing the venous and arterial anastomoses in small animals. Furthermore, the time required for flap fixation is quite short in this model. Therefore, we anchored the flap to the zygomaticus and masseter muscles of the recipient first, and vessel anastomoses were performed subsequently.

We have not performed nerve repair in this rat model because of the small size of nerves and difficulties in access. In fact, there was no need for nerve repair because the composite flap was com- 
posed of skin, subcutaneous tissue, and ear with cartilage, without any of muscles needed for facial animation. In humans, however, the anatomic structures are more suitable for performing nerve repair, and it is important to achieve facial muscle innervation for optimal functional results [10].

Another critical factor for successful CTA is immunosuppressive therapy. CTAs are considered highly immunogenic mainly because of the skin component. Therefore, recipients require lifelong immunosuppression treatment with higher levels of immunosuppressive agents than those in organ allograft patients [11]. However, continuous high-dose immunosuppressive therapy frequently causes serious complications, such as nephrotoxicity and hepatotoxicity [12]. In our model, a low-dose cyclosporine A monotherapy protocol was used. Cyclosporine $\mathrm{A}$ is a calcineurin inhibitor that acts via suppression of T-cell mediated interleukin-2 production [13]. Previous reports on low-dose cyclosporine A therapy in CTA models did not confirm long-term survival of composite tissue transplants [14]. Siemionow et al. modified this approach by applying a high induction dose of cyclosporine A, which was then reduced to a low maintenance dose. Under this modified protocol, all the hemifacial allotransplantation recipients survived without any sign of toxicity for up to 240 days after transplantation [7].

In summary, we described a rat hemifacial allograft transplantation model and its benefits. Overall, the hemifacial transplantation model is a convenient, cost-effective, and reproducible model, which is suitable for training craniofacial reconstructive surgeons who are not familiar with CTA procedures. This model has some limitations. The rat is too small to resemble a human patient in terms of anatomic and physiologic characteristics. In addition, this model provides only simplified versions of flap harvesting and transferring procedures that craniofacial reconstructive surgeons might face in the future, and does not require advanced microsurgery skills. Therefore, it is important to develop experimental CTA models resembling human and to improve operative techniques.

\section{REFERENCES}

1. Siemionow MZ, Kulahci Y, Bozkurt M. Composite tissue allotransplantation. Plast Reconstr Surg 2009;124:e327-39.

2. Hong JW, Kim YS, Yun IS, Lee DW, Lee WJ, Roh TS, Lew DH, Kim YO, Rah DK, Tark KC, Park BY. Current status of face transplantation: where do we stand in Korea? Arch Craniofac Surg 2012;13:85-94.

3. Ulusal BG, Ulusal AE, Ozmen S, Zins JE, Siemionow MZ. A new composite facial and scalp transplantation model in rats. Plast Reconstr Surg 2003;112:1302-11.

4. Nie C, Yang D, Li N, Liu G, Guo T. Establishing a new orthotopic composite hemiface/calvaria transplantation model in rabbits. Plast Reconstr Surg 2008;122:410-8.

5. Shengwu Z, Qingfeng L, Hao J, Banich J, Kaiding F, Benson C, Huiyong W, Danning Z, Bing G, Qinxiu L, Lujia T, Tao Z, Yuping L, Tisheng Z. Developing a canine model of composite facial/scalp allograft transplantation. Ann Plast Surg 2007;59:185-94.

6. Cendales LC, Xu H, Bacher J, Eckhaus MA, Kleiner DE, Kirk AD. Composite tissue allotransplantation: development of a preclinical model in nonhuman primates. Transplantation 2005;80:1447-54.

7. Demir Y, Ozmen S, Klimczak A, Mukherjee AL, Siemionow M. Tolerance induction in composite facial allograft transplantation in the rat model. Plast Reconstr Surg 2004;114:1790-801.

8. Siemionow M, Gozel-Ulusal B, Engin Ulusal A, Ozmen S, Izycki D, Zins JE. Functional tolerance following face transplantation in the rat. Transplantation 2003;75:1607-9.

9. Siemionow M, Unal S, Agaoglu G, Sari A. A cadaver study in preparation for facial allograft transplantation in humans: part I. What are alternative sources for total facial defect coverage? Plast Reconstr Surg 2006;117:864-72.

10. Kim CW, Do ER, Kim HT. A new facial composite flap model (panorama facial flap) with sensory and motor nerve from cadaver study for facial transplantation. J Korean Cleft Palate-Craniofac Assoc 2011;12:86-92.

11. Whitaker IS, Duggan EM, Alloway RR, Brown C, McGuire S, Woodle ES, Hsiao EC, Maldonado C, Banis JC Jr, Barker JH. Composite tissue allotransplantation: a review of relevant immunological issues for plastic surgeons. J Plast Reconstr Aesthet Surg 2008;61:481-92.

12. Gorantla VS, Barker JH, Jones JW Jr, Prabhune K, Maldonado C, Granger DK. Immunosuppressive agents in transplantation: mechanisms of action and current anti-rejection strategies. Microsurgery 2000;20:420-9.

13. Kim SK, Aziz S, Oyer P, Hentz VR. Use of cyclosporin A in allotransplantation of rat limbs. Ann Plast Surg 1984;12:249-55.

14. Inceoglu S, Siemionow M, Chick L, Craven CM, Lister GD. The effect of combined immunosuppression with systemic low-dose cyclosporin and topical fluocinolone acetonide on the survival of rat hind-limb allografts. Ann Plast Surg 1994;33:57-65. 